\title{
Éclosion de COVID-19 dans un établissement de services personnels à Kingston, Ontario en 2020
}

\author{
Anthony $\mathrm{Li}^{1,2}$, Stéphanie Parent ${ }^{1,2}$, Azim Kasmani ${ }^{1,3}$, T Hugh Guan $^{1,3}$, Kieran Moore ${ }^{1,3 *}$
}

\section{Résumé}

Contexte : Pendant la pandémie de maladie à coronavirus 2019 (COVID-19), I'Ontario a créé un plan de réouverture de l'économie en trois phases. Des éclosions étaient attendues à chaque phase. Une semaine après la phase deux de réouverture dans la région de l'administration provinciale de santé publique de de Kingston, Frontenac, Lennox et Addington, un cas positif a été signalé après trois semaines sans nouveau cas de COVID-19. L'objectif de ce rapport est de décrire cette éclosion de la COVID-19, déclarée dans un établissement de services personnels, et l'intervention de la santé publique pour contenir cette éclosion.

Méthodes : L'enquête sur l'éclosion a porté sur tous les cas de COVID-19 survenus dans la région de Kingston, Frontenac, Lennox et Addington entre le 20 juin 2020 et le 3 juillet 2020. Des inspecteurs et des infirmières en Santé publique ont été rapidement déployés pour inspecter l'ESP. Une approche multimodale des dépistages à haut volume a impliqué des centres d'évaluation fixes, des capacités de dépistage au volant et des dépistages ciblés sur le site de l'éclosion. Les tests de dépistage ont été effectués par une amplification en chaîne par polymérase en temps réel au laboratoire local de Santé publique Ontario.

Résultats : Trente-sept cas ont été liés à cette éclosion : $38 \%$ par exposition directe à l'ESP; $32 \%$ par contact familial; et $30 \%$ par contact social et professionnel. Un événement de supercontamination a contribué à $38 \%$ du total des cas. La majorité des cas se situaient dans les quintiles inférieurs ou moyens lorsque l'on analysait la difficulté matérielle. Les taux de dépistage ont été multipliés par quatre par rapport aux semaines de référence précédentes en réponse aux messages médiatiques et aux messages de Santé publique, ce qui a entraîné un faible pourcentage de positivité.

Conclusion : L'interaction entre les dépistages intensifs et accessibles, la rapidité d'exécution en laboratoire, la recherche des contacts dans les 24 heures suivant l'obtention de résultats de laboratoire positifs conformément aux normes provinciales, une communication publique fréquente, les inspections rapides, l'auto-isolement et le port obligatoire du masque ont permis de stopper l'éclosion. Des inspections ou des autovérifications doivent être exigées dans tous les ESP avant la réouverture et les responsables de la gestion des éclosions doivent travailler avec les ESP pour réduire la possibilité d'événements de supercontamination.
Cette oeuvre est mise à la disposition selon les termes de la licence internationale Creative Commons Attribution 4.0

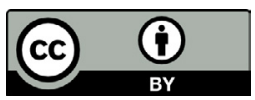

Affiliations

${ }^{1}$ Kingston, Frontenac, Lennox \& Addington (KFL\&A) Public Health, Kingston, ON

2 École de Médicine, Université Queen's, Kingston, ON

${ }^{3}$ Programme de résidence en santé publique et en médecine préventive, Université Queen's, Kingston, ON

*Correspondance :

kieran.moore@kflaph.ca

Citation proposée : Li A, Parent S, Kasmani A, Guan TH, Moore K. Éclosion de COVID-19 dans un établissement de services personnels à Kingston, Ontario en 2020. Relevé des maladies transmissibles au Canada 2021;47(4):236-44. https://doi.org/10.14745/ccdr.v47i04a06f

Mots-clés : COVID-19, SRAS-CoV-2, éclosion, service personnel, santé publique, prévention et contrôle de I'infection, gestion des cas et des contacts, événement de supercontamination

\section{Introduction}

Le coronavirus du syndrome respiratoire aigu sévère 2

(SRAS-CoV-2) est un bêtacoronavirus à acide ribonucléique (ARN) enveloppé qui provoque une maladie à coronavirus (COVID-19), dont les symptômes courants sont la fièvre, la toux,
I'essoufflement et la fatigue $(1,2)$. Les cas de COVID-19 sévères peuvent nécessiter une admission en unité de soins intensifs et peuvent potentiellement entraîner la mort (3). Le SRAS-CoV-2 se transmet le plus souvent par contact étroit par des gouttelettes 
liquides émises par des personnes infectées et a été classé avec d'autres nouveaux coronavirus comme maladie transmissible à déclaration obligatoire en Ontario le 22 janvier $2020(4,5)$.

Alors que la pandémie de COVID-19 a entraîné 100000 cas positifs et près de 9000 décès au Canada en date de juillet 2020, la région de Kingston, Frontenac, Lennox et Addington a connu une très faible incidence communautaire de COVID-19 (6). Bien que la région de Kingston, Frontenac, Lennox et Addington compte 209023 habitants, dont $20 \%$ sont âgés de plus de 65 ans, aucun décès n'a été attribué à cette maladie, un seul cas a été enregistré dans un établissement de soins de longue durée et 63 cas ont été recensés avant cette éclosion (7).

Le gouvernement de l'Ontario a fourni un plan de réouverture de I'économie en trois phases (8). La région de Kingston, Frontenac, Lennox et Addington a été autorisée à passer de la phase 1 à la phase 2 le 12 juin 2020, ce qui a permis la réouverture des établissements de services personnels tels que les salons de manucure, les barbiers et les salons de tatouage. Bien que les établissements de services personnels aient reçu des conseils et été mis au courant des pratiques exemplaires, aucune inspection n'était requise avant la réouverture. Après une période de trois semaines sans nouveaux cas de COVID-19 dans la région de Kingston, Frontenac, Lennox et Addington, un cas positif a été détecté le 20 juin 2020 chez un membre du personnel hospitalier, une semaine après la phase deux de la réouverture. Deux autres cas positifs ont été signalés trois jours plus tard, mais aucun n'avait de lien avec l'hôpital. Grâce à des entretiens répétés et des analyses de réseaux sociaux, les enquêteurs ont identifié un salon de manucure comme étant la source commune. Le service de Santé publique de la région de Kingston, Frontenac, Lennox et Addington a envoyé une équipe d'inspecteurs/ inspectrices et d'infirmiers/infirmières pour enquêter sur les pratiques de prévention et de contrôle des infections et pour effectuer un dépistage des travailleurs. Six travailleurs ont reçu un résultat positif de COVID-19 et une éclosion a été déclarée le 25 juin 2020.

Cette éclosion démontre comment les établissements de services personnels peuvent contribuer à la transmission des maladies. Des inspections ou des autovérifications avec un mécanisme de déclaration doivent être exigées dans tous les établissements de services personnels avant leur réouverture et doivent être considérées comme des sources potentielles d'infection lors des enquêtes sur les cas et les contacts. Une intervention régionale collaborative entre la communauté, l'agence de santé publique locale, les laboratoires et les hôpitaux qui gèrent les centres d'évaluation a permis une gestion rapide de l'éclosion. L'objectif de ce rapport est de décrire l'enquête qui a conduit à la découverte de la source de l'éclosion et les interventions pour contenir l'éclosion.

\section{Méthodes}

\section{Détection de l'éclosion}

Le cas index de cette éclosion, signalé le 20 juin 2020, était un membre du personnel hospitalier. Les enquêtes initiales n'ont pas permis de découvrir une source parmi les contacts professionnels ou familiaux. Le 23 juin, deux autres cas positifs ont été déterminés. Aucun des trois n'a déclaré avoir des contacts entre eux ni n'a trouvé de contacts communs. Lors d'entretiens répétés par les enquêteurs, un lien commun a été découvert : les trois cas s'étaient rendus dans le même salon de manucure la semaine du 14 juin. Le service de Santé publique de la région de Kingston, Frontenac, Lennox et Addington a rapidement déployé un inspecteur de santé publique et une équipe d'infirmières sur place pour enquêter sur l'établissement de services personnels, effectuer un test de dépistage à tous les travailleurs et examiner les pratiques de prévention et de contrôle des infections. Un travailleur a reçu un résultat de dépistage positif à la COVID-19 dans les 48 heures, et six travailleurs au total ont finalement reçu un résultat positif, ce qui démontre qu'il y a une très forte chance que ce lieu était la source de l'éclosion. Le 25 juin, le service de Santé publique de la région de Kingston, Frontenac, Lennox et Addington a officiellement déclaré l'éclosion au public et a ordonné la fermeture du salon de manucure jusqu'à nouvel ordre.

\section{Enquête sur le cas}

Les cas ont été déterminés par un test de dépistage positif de réaction en chaîne par polymérase ( $P C R$ ) en temps réel, conformément à la définition du ministère provincial (9). La définition de cas interne utilisée par le service de Santé publique de la région de Kingston, Frontenac, Lennox et Addington pour cette éclosion était un client ou un contact proche d'un client positif du salon de manucure. On a supposé que le risque de transmission a commencé à la date d'ouverture officielle de l'établissement de services personnels, le 12 juin 2020. Les personnes dont le test de dépistage de la COVID-19 était positif ont été contactées dans les 24 heures suivant leur résultat par le personnel du service de Santé publique de la région de Kingston, Frontenac, Lennox et Addington, conformément aux directives provinciales (10). L'enquête sur la gestion des cas et des contacts comprenait la date d'apparition, les symptômes, les antécédents d'exposition, y compris les voyages ou les contacts positifs, les facteurs de risque et tout contact proche (avec le niveau de risque d'exposition). Tous les cas ont fait l'objet d'une surveillance active pendant une période de 14 jours, conformément aux directives du ministère de la Santé de l'Ontario (11). Le coût moyen estimé par cas était de $400 \$$ et nécessitait sept heures de travail de la part du personnel, tandis que le coût moyen par contact était de 160 \$ et nécessitait trois heures de travail de la part du personnel.

\section{Enquête de laboratoire}

Le site de Kingston du Laboratoire de Santé publique Ontario a effectué des tests de dépistage de PCR en temps réel sur des échantillons et a signalé des cas en suivant la définition de cas de 
la COVID-19 établie par le ministère de la Santé. Un cas confirmé est défini comme « une personne dont le laboratoire a confirmé I'infection à la COVID-19 à l'aide d'un test de dépistage validé, consistant en un test de dépistage positif d'amplification de l'acide nucléique sur au moins une cible génomique précise » (9). Le Laboratoire de Santé publique Ontario a utilisé l'essai du gène de l'enveloppe $(E)$ comme cible génomique pour son test dépistage développé en laboratoire. Le temps d'exécution du test de dépistage de la COVID-19 était de 24 à 48 heures.

\section{Analyse des données}

Le logiciel Microsoft ${ }^{\mathrm{MC}}$ Excel 2016 a été utilisé pour créer la courbe épidémique et les analyses descriptives, tandis qu'une analyse du réseau social a été réalisée à l'aide de l'outil SocNetV 2.5. Une analyse spatiale a été réalisée à l'aide de systèmes d'information géographique pour construire une carte choroplèthe afin de déterminer les secteurs de recensement à forte incidence.

Les taux d'attaque bruts pour les générations suivantes ont été calculés à partir du nombre de cas confirmés par génération et du nombre de personnes susceptibles d'être contaminées (12). Les personnes susceptibles d'être contaminées ont été définies comme des contacts à haut risque, les contacts n'étant comptés qu'une seule fois s'ils sont partagés entre les cas. Les données d'écouvillonnage des centres d'évaluation et les données de cas du Laboratoire de Santé publique Ontario ont été utilisées pour calculer le pourcentage de positivité.

Le score de difficulté matérielle mesure l'incapacité des personnes à accéder aux besoins matériels de base tels que le niveau d'éducation ou un logement de qualité (13). Des indices de difficulté ont été déterminés pour chaque cas afin d'établir un profil complet de difficulté matérielle comme indicateur du statut socio-économique. Les profils selon la difficulté matérielle ont été regroupés en quintiles, les scores des quintiles les plus élevés indiquant un niveau de difficulté plus important.

\section{Interventions}

La liste de contacts des clients fournie par le salon de manucure était incomplète, ce qui empêchait une gestion efficace de ceux-ci. Ainsi, le 25 juin 2020, le service de Santé publique de la région de Kingston, Frontenac, Lennox et Addington a publié une annonce publique demandant à tous les clients de cet établissement de services personnels entre le 12 et le 24 juin de faire un dépistage de la COVID-19 et de s'auto-isoler pendant 14 jours à partir de la date de leur dernière visite. Un certain nombre d'entreprises ont volontairement fermé par mesure de précaution, leurs employés ayant visité l'établissement de services personnels et ayant reçu des résultats positifs au test de dépistage.

En plus du salon de manucure initial, quatre employés de deux autres salons de manucure ont finalement reçu des résultats positifs au test de dépistage de la COVID-19 par contact avec le personnel du premier salon. Le service de Santé publique de la région de Kingston, Frontenac, Lennox et Addington a publié un communiqué de presse demandant à tous les clients qui ont fréquenté ces deux établissements de services personnels de s'auto-isoler et de faire un test de dépistage. Les deux salons ont été fermés jusqu'à nouvel ordre. Plus de 500 clients se sont rendus dans le premier salon de manucure associé à l'éclosion entre le 12 et le 20 juin, et tous ont été invités à s'auto-isoler et à faire un test de dépistage. On a également demandé à 180 autres contacts proches des cas positifs de s'auto-isoler et de faire un test de dépistage.

Afin de minimiser les risques d'éclosions supplémentaires dans les lieux publics, le médecin-hygiéniste de la région de Kingston, Frontenac, Lennox et Addington a émis une ordonnance le 27 juin 2020, en vertu de l'article 22 de la Loi sur la protection et la promotion de la santé de l'Ontario, interdisant aux personnes d'entrer et de rester à l'intérieur des établissements commerciaux intérieurs si elles ne portent pas un couvre-visage et assurant également la disponibilité de rince-mains à base d'alcool à toutes les entrées et sorties. En outre, le 30 juin, le service de Santé publique de la région de Kingston, Frontenac, Lennox et Addington a imposé l'auto-isolement et la quarantaine aux cas de COVID-19 ou aux contacts proches d'un cas positif. Un avis a également été publié demandant à tous les établissements de services personnels de remplir une liste de contrôle conçue pour assurer la conformité des établissements de services personnels avec les règlements de relatif aux pratiques de prévention et de contrôle des infections (14).

\section{Résultats}

Il y a eu 37 cas de COVID-19 liés à l'éclosion entre le 20 juin et le 3 juillet (figure 1). La majorité des cas (65\%) étaient des femmes et l'âge moyen était de 38,6 ans dans plage allant de 11 mois à 69 ans. Les cas concernaient diverses professions, notamment dans les domaines de la santé, de l'éducation, de la construction, de la restauration, du commerce de détail et des services correctionnels. Sur les 37 cas, 14 (38\%) résultaient d'une exposition directe à l'établissement de services personnels, 12 (32\%) étaient des contacts familiaux avec un autre cas, et les 11 autres (30\%) étaient des contacts sociaux et professionnels avec un cas (figure 2). Un client de l'établissement de services personnels a infecté à lui seul six personnes lors d'une réunion sociale et deux collègues de travail.

Une fois que le service de Santé publique de Kingston, Frontenac, Lennox et Addington a identifié le salon de manucure comme étant le lien commun entre les trois premiers cas, un inspecteur en santé publique et une équipe et $d$ 'infirmières ont été rapidement déployés sur place pour enquêter. Des lacunes considérables dans les pratiques de prévention et de contrôle des infections ont été constatées. On a observé que 
Figure 1 : Courbe épidémique pour l'éclosion de COVID-19 dans un établissement de services personnels de la région de Kingston, Frontenac, Lennox et Addington, $2020(n=37)$

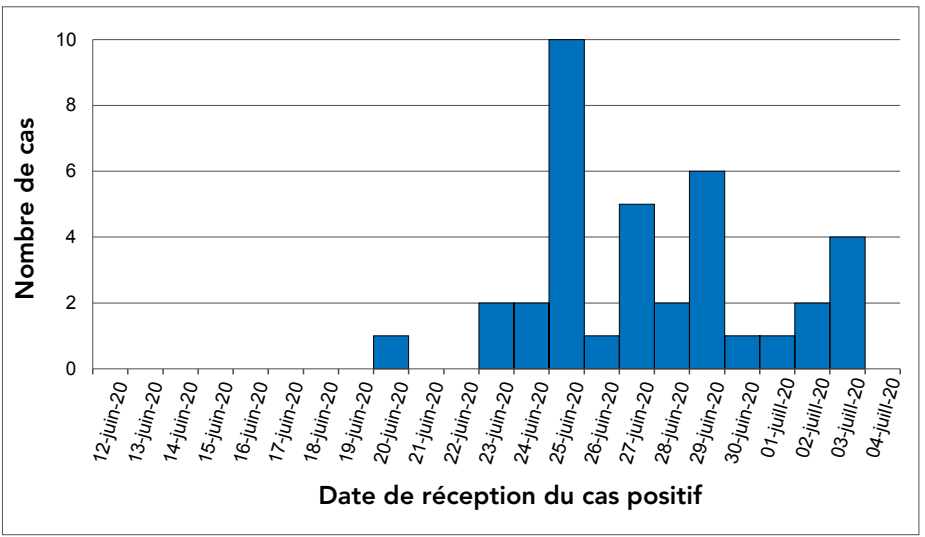

Figure 2 : Analyse du réseau social pour les cas de COVID-19 dans l'établissement de services personnels de la région de Kingston, Frontenac, Lennox et Addington, 2020

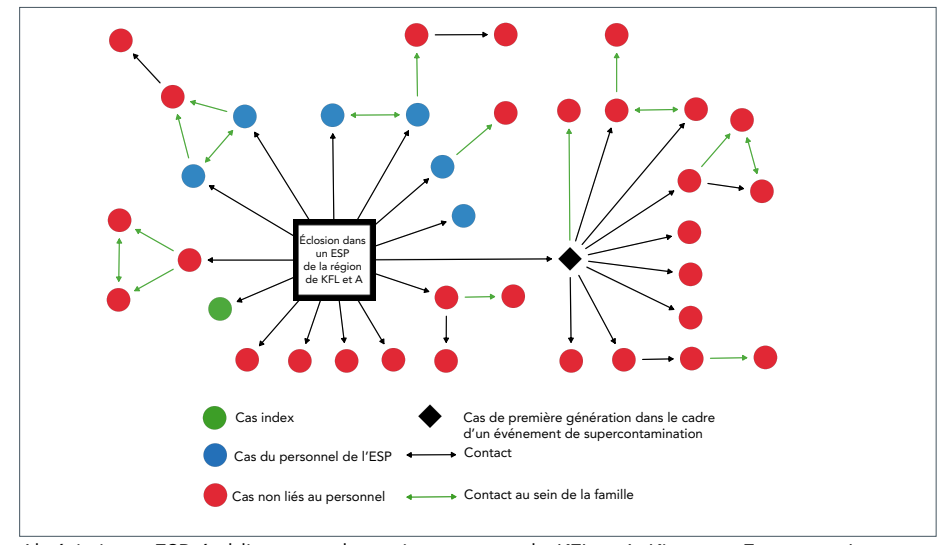

Abréviations : ESP, établissement de services personnels; KFL et A, Kingston, Frontenac, Lennox et Addington

le personnel avait une hygiène des mains inadéquate, se lavant seulement pendant quelques secondes et sans utiliser de savon. Le personnel n'a pas subi de dépistage des symptômes de la COVID-19 et s'est présenté au travail avec des symptômes. Des clients et des membres du personnel ont également observé que les masques faciaux étaient mal portés, notamment, ils pendaient sous le menton, exposaient le nez ou étaient retirés pour utiliser un téléphone. Les postes de travail et les équipements n'étaient pas nettoyés avant d'être désinfectés, et les surfaces à contacts fréquents n'étaient nettoyées et désinfectées qu'une fois par jour. En outre, la consignation manuscrite des noms et des numéros de téléphone des clients était souvent incomplète ou illisible. Six travailleurs ont finalement reçu des résultats positifs au test de dépistage de la COVID-19.
Sept (19\%) des 37 cas étaient asymptomatiques au moment du diagnostic. Trois des sept cas asymptomatiques n'ont jamais présenté de symptôme de COVID-19. Un cas de transmission de la COVID-19 a été déterminé à partir d'un véritable cas asymptomatique. Un seul cas associé à l'éclosion a été hospitalisé. Cette personne présentait des comorbidités, ce qui a nécessité une admission de huit jours à l'hôpital et un séjour de cinq jours dans l'unité de soins intensifs. Aucun décès n'a été associé à cette éclosion.

Le profilage de la carte choroplèthe a indiqué un nombre de cas plus élevé plus près de la source de l'éclosion et une distribution spatiale relativement uniforme dans les secteurs de recensement (figure 3). Les profils selon la difficulté matérielle des cas indiquent que $54 \%$ des cas se situent dans les quintiles inférieurs à moyens de difficulté matérielle, associés à des zones de statut socio-économique plus élevé, tandis que $38 \%$ des cas se situent dans les quintiles supérieurs de difficulté matérielle, associés à des zones de statut socio-économique plus faible (figure 4). Aucune donnée n'était disponible pour les $8 \%$ de cas restants.

Figure 3 : Carte choroplèthe des cas de COVID-19 associés à l'établissement de services personnels de la région de Kingston, Frontenac, Lennox et Addington 2020

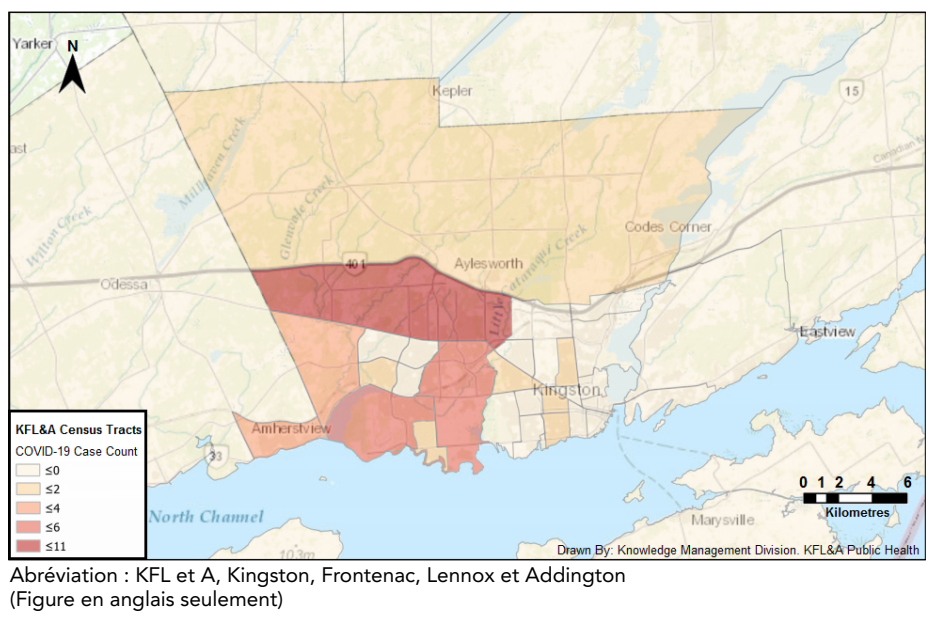

Figure 4 : Profil selon la difficulté matérielle des cas associés à l'éclosion de l'établissement de services personnels de la région de Kingston, Frontenac, Lennox et Addington, 2020

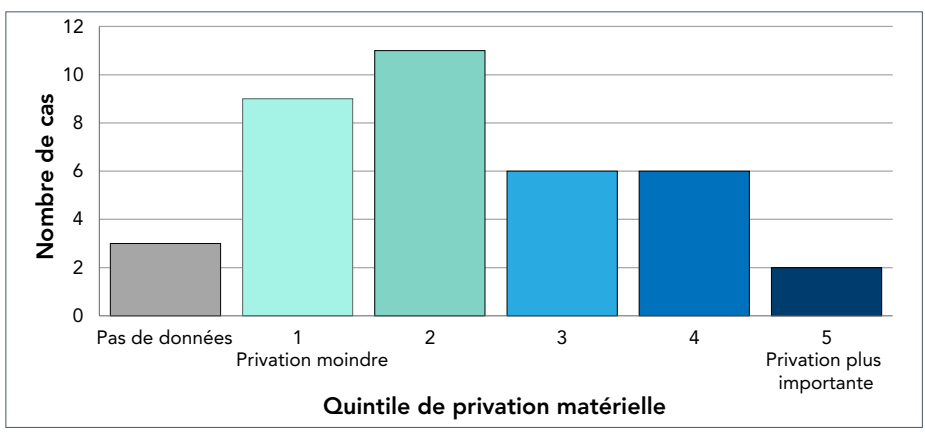


Quatre générations de transmission ont été déterminées (figure 5). Il y a eu 14 cas dans la première génération dans ce qui a été estimé à 500 clients et membres du personnel qui ont été potentiellement exposés à la COVID-19. En raison de dossiers incomplets sur les clients, une estimation a été nécessaire et a été calculée en utilisant le nombre probable de clients servis, chaque jour, sur la base de la capacité du salon pendant les 13 jours où il était ouvert. Ainsi, le taux d'attaque de la première génération était de $2,8 \%$. La deuxième génération de 16 cas et 101 contacts a eu un taux d'attaque de 15,8 \%. La troisième génération, composée de six cas et de 49 contacts a eu un taux d'attaque de $12,2 \%$. La quatrième génération, composée d'un cas et de 14 contacts, a eu un taux d'attaque de $7,4 \%$. Les coûts bruts estimés pour la gestion des cas et des contacts de cette enquête sur l'éclosion ont été d'environ 41040 \$ pour 751 heures de travail.

Figure 5 : Cas de COVID-19 et contacts par génération à partir de l'établissement de services personnels de la région de Kingston, Frontenac, Lennox et Addington, 2020

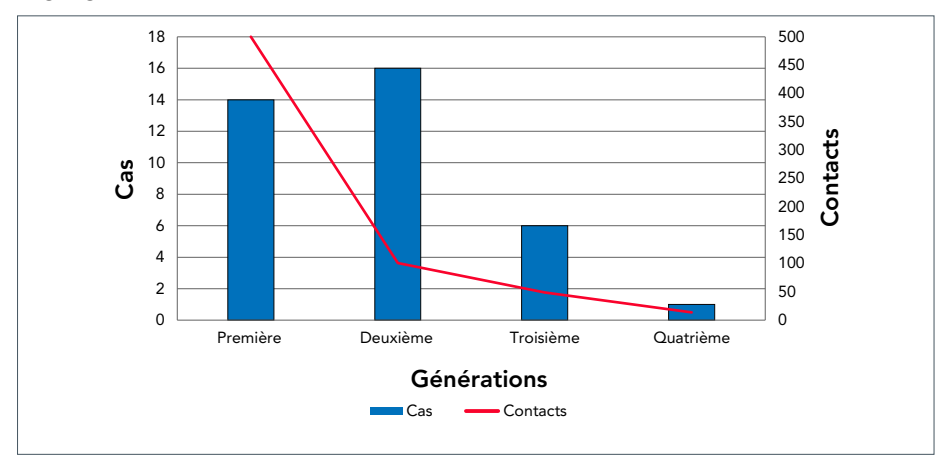

Le service de Santé publique de la région de Kingston,

Frontenac, Lennox et Addington avait une approche multimodale d'écouvillonnage, comprenant des centres d'évaluation fixes, une capacité de dépistage au volant et des tests de dépistage ciblés sur le site l'établissement. Les taux de dépistage entre le 20 juin et le 5 juillet ont été élevés en réaction à l'attention médiatique et aux messages du service de Santé publique de la région de Kingston, Frontenac, Lennox et Addington. Le nombre de tests de dépistage effectués pendant l'éclosion a été multiplié par approximativement quatre par rapport aux semaines de référence précédentes (figure 6). En outre, le nombre cumulé de tests de dépistage effectués dans la région de Kingston, Frontenac, Lennox et Addington depuis le début de cette éclosion a dépassé les 10000 tests le 7 juillet. Le pourcentage de positivité est resté faible et a atteint un sommet de 0,61\% le 29 juin (figure 7).
Figure 6 : Nombre de patients soumis à des prélèvements par jour dans les centres d'évaluation

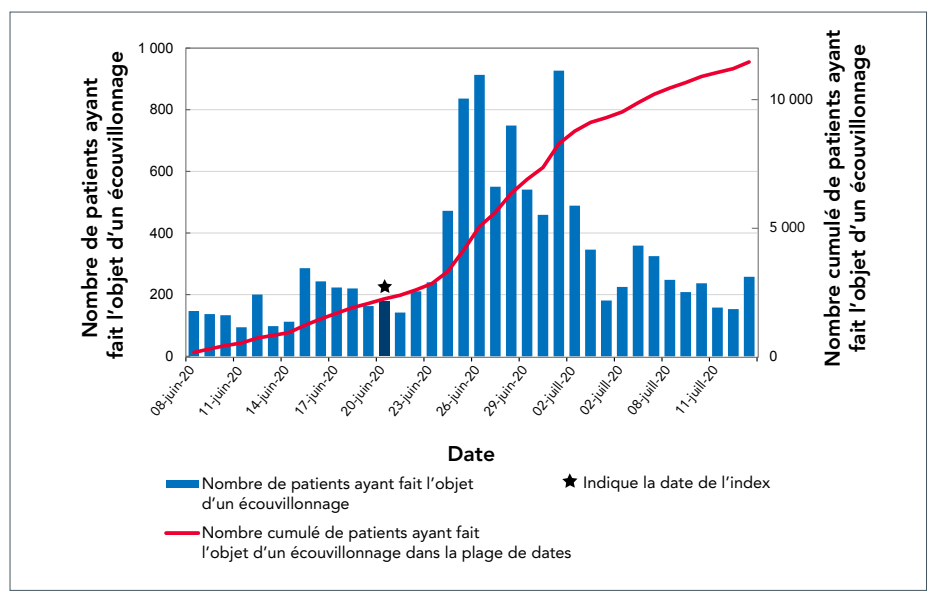

Figure 7 : Pourcentage de positivité moyenne mobile sur 7 jours des tests de dépistage de la COVID-19

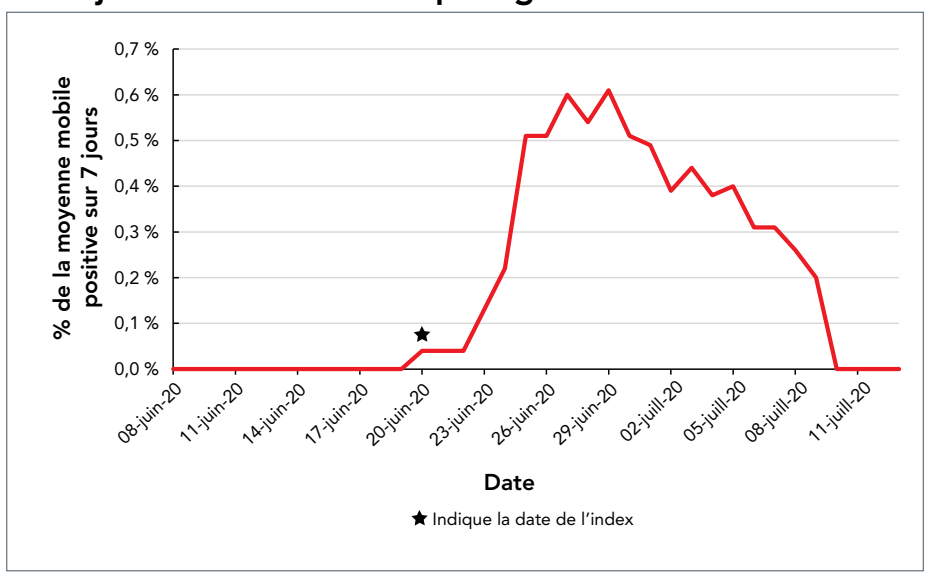

\section{Discussion}

Avant cette éclosion dans l'établissement de services personnels, la région de Kingston, Frontenac, Lennox et Addington avait I'un des taux de cas les plus bas de l'Ontario avec 29,1 cas cumulés pour 100000 habitants (15). Après trois semaines sans nouveaux cas de COVID-19 dans la région de Kingston, Frontenac, Lennox et Addington, la détection d'une éclosion une semaine après la phase deux de réouverture a révélé des lacunes dans le respect des recommandations et des meilleures pratiques émises par le gouvernement.

La transmission des maladies est facilitée dans un établissement de services personnels en raison des difficultés de maintien d'une distance physique lors de la réception d'un service. Le personnel peut être en contact étroit avec des dizaines de clients chaque jour et toute réutilisation d'équipements insuffisamment stérilisés aggrave la situation. La transmission 
de mycobactéries, du virus de l'hépatite $B$ et du virus de I'hépatite $\mathrm{C}$ a été bien documentée dans les établissements de services personnels (16-19). Une combinaison de facteurs a contribué à cette éclosion dans l'établissement de services personnels. Une hygiène des mains insuffisante, une utilisation inadéquate du couvre-visage et un personnel travaillant alors qu'il présentait des symptômes ont permis la transmission de la COVID-19. En plus de déficiences concernant le nettoyage des postes de travail et des équipements, le lavage des mains pendant seulement quelques secondes sans utilisation de savon et le port d'un couvre-visage qui exposait la bouche ou le nez ont indiqué de mauvaises pratiques de prévention et de contrôle des infections. L'efficacité d'une bonne adhésion aux pratiques de prévention et de contrôle des infections a été constatée dans un établissement de services personnels du Missouri, aux États-Unis, où deux coiffeurs présentant des symptômes infectieux (COVID-19) n'ont transmis le virus à aucun de leurs 139 clients exposés, car tout le personnel et les clients portaient correctement des couvre-visages et suivaient des pratiques rigoureuses d'hygiène des mains (20). Une inspection du respect des meilleures pratiques des pratiques de prévention et de contrôle des infections avant la réouverture est essentielle pour prévenir de futures éclosions. Une autre approche pourrait être une autovérification des pratiques de prévention et de contrôle des infections et un mécanisme de déclaration à santé publique.

Les 37 cas de COVID-19 liés au salon de manucure ont nécessité des mesures rigoureuses de la part de la santé publique locale pour contenir la propagation du virus, y compris la détermination rapide de la source de l'éclosion, une large sensibilisation de la communauté, des initiatives de dépistage à haut volume et la détermination des cas ultérieurs. Cela a permis de rechercher rapidement les contacts dans le cadre des directives provinciales de 24 heures et d'isoler les cas et les contacts pour contenir l'éclosion. L'efficacité a pu être constatée par la diminution des taux d'attaque après la deuxième génération, indiquant que les cas et les contacts s'isolaient et empêchaient toute nouvelle transmission (figure 5).

Sept (19\%) cas étaient asymptomatiques au moment du diagnostic, et trois ( $8 \%$ ) sont restés asymptomatiques. Ce chiffre se situe dans la partie inférieure de la fourchette de $18 \%$ à $57 \%$ des taux d'asymptomatologie précédemment rapportés dans la littérature (21-24). Ces cas ont été découverts grâce à un volume élevé d'écouvillonnages et de tests de dépistage, ce qui a entraîné un faible pourcentage de positivité tout au long de l'éclosion. La détection précoce des cas asymptomatiques a permis de les isoler à temps pour éviter toute transmission ultérieure (25). Les personnes asymptomatiques sont difficiles à repérer, moins susceptibles de s'auto-isoler et plus susceptibles d'avoir des contacts sociaux (26). Cette éclosion confirme que les cas qui ne présentent pas de symptôme initialement développent souvent des symptômes et deviennent des cas présymptomatiques. Des preuves supplémentaires sont nécessaires pour mieux comprendre la prévalence et le rôle de la transmission asymptomatique réelle par rapport à la transmission présymptomatique de la COVID-19.

La transmission secondaire aux personnes qui ne fréquentaient pas le salon de manucure s'est faite principalement par contacts familiaux. Au total, 12 (32\%) cas étaient des contacts familiaux avec un cas de l'éclosion. Des rapports antérieurs ont montré des taux d'attaque secondaires de la COVID-19 dans les familles $11,2 \%$ à $35 \%$ (27-31). Ce type de transmission était attendu, ayant été décrit dans les cas de SRAS-CoV, du syndrome respiratoire du Moyen-Orient (SRMO) et de l'influenza (32-34). Les personnes vivant sous le même toit pratiquent généralement des activités qui facilitent l'infection par les gouttelettes, comme I'intimité et le partage de nourriture et de boissons. Bien qu'il puisse être difficile d'interrompre la transmission du SRAS-CoV-2 entre les contacts familiaux, la gestion de cette éclosion a montré qu'il était plus facile de limiter la propagation en dehors de la famille en déterminant les cas au début de la maladie et en les isolant ainsi qu'en isolant les contacts proches.

Les événements de supercontamination se produisent lorsqu'un cas propage la maladie beaucoup plus largement que les autres. Ces événements ont été constatés dans le monde entier, notamment dans une chorale aux États-Unis, une boîte de nuit en Corée du Sud et une cérémonie de bénédiction en Chine (35-37). Un événement de supercontamination a été observé dans cette éclosion à l'établissement de services personnels : un client était responsable de 14 (38\%) cas, dont aucun ne fréquentait l'institut de manucure. Parmi ceux-ci, deux étaient des contacts sur le lieu de travail, tandis que six ont participé à un événement social avec le cas de première génération, transmettant ensuite le virus à leurs propres contacts. La recherche des contacts pour ce groupe a été très difficile : un certain nombre de contacts n'ont pas été identifiés lors de l'entretien initial du premier cas. Cependant, les personnes qui ont reçu un résultat positif par la suite ont déclaré avoir eu des contacts étroits avec le cas initial. Les événements de supercontamination ont joué un rôle important dans la transmission de la COVID-19. Une récente étude de modélisation a proposé qu'un moyen efficace de contrôler l'épidémie était de limiter les contacts aléatoires en dehors des lieux de travail et des milieux familiaux (35). Cela va dans le sens des directives provinciales en vigueur au début de l'éclosion, qui préconisent de maintenir un cercle social d'un maximum de 10 personnes (38). Le client de l'événement de supercontamination de la région de Kingston, Frontenac, Lennox et Addington avait un cercle social bien plus grand que 10 personnes, ce qui confirme l'importance de maintenir de petits cercles sociaux pour éviter les événements de superproduction.

La mise en quarantaine massive de centaines de personnes a eu de vastes répercussions sociales, sanitaires et économiques. 
Le lendemain de l'annonce de l'éclosion, des centaines de personnes ont fait la queue devant le centre d'évaluation, certaines attendant pendant des heures pour avoir un test de dépistage. Les entreprises ont fermé en raison de la nécessité de mettre les travailleurs en quarantaine, et la communauté a hésité à accéder aux entreprises restées ouvertes. En Ontario, les régions dont le statut socio-économique est le plus bas ont supporté la plus grande charge de COVID-19 (39). Cependant, la majorité des cas associés à cette éclosion étaient situés dans des zones géographiques associées à un statut socio-économique plus élevé. Il est possible que l'impact plus important sur les personnes ayant un statut socio-économique élevé soit dû à leur plus grande probabilité de pouvoir acheter des services esthétiques. La carte choroplèthe $n$ 'indique aucune association majeure au-delà de la proximité de la source de l'éclosion.

\section{Limites}

Le présent rapport comporte plusieurs limites. Les données sur les symptômes et les contacts proches étaient autodéclarées et vulnérables aux biais de désirabilité sociale et de rappel. Compte tenu de la couverture médiatique et de la stigmatisation entourant les activités sociales, les répondants peuvent avoir fourni des déclarations jugées plus acceptables socialement ce qui s'avère une tendance commune dans la gestion des cas et des contacts (40-42). Certaines des données peuvent être incomplètes en raison des limitations des ressources et du système de gestion des données. Les centres d'évaluation, le Laboratoire de Santé publique Ontario, I'agence locale de santé publique et les hôpitaux utilisent différents systèmes d'information électroniques, qui ne sont pas intégrés. En outre, l'augmentation soudaine et importante du nombre de cas et de contacts a partiellement submergé les centres d'évaluation, entraînant des problèmes potentiels de qualité des données. De plus, il n'est pas possible de déterminer une relation de cause à effet entre une initiative unique et son effet sur le contrôle de l'éclosion, car toutes les initiatives ont probablement fait en sorte qu'un travail conjoint se soit effectué pour limiter l'éclosion. En outre, les déductions fondées sur des indicateurs géographiques peuvent être erronées et ne pas s'appliquer au niveau individuel un traînant un sophisme écologique. Enfin, les données n'ont été recueillies que sur les cas et les contacts au sein de la compétence de la région de Kingston, Frontenac, Lennox et Addington, les personnes ne relevant pas de cette compétence étant renvoyées à leur agence de Santé publique locale respective. Ainsi, les données les concernant peuvent ne pas avoir été saisies, ce qui entraîne une sous-déclaration. Malgré ces limites, ce rapport ajoute à la littérature en examinant une éclosion de COVID-19 dans un établissement de services personnels et en décrivant les initiatives ultérieures qui ont conduit à son interruption dans une agence de santé publique locale de taille moyenne en Ontario.

\section{Conclusion}

Dans l'ensemble, la Santé publique de la région de Kingston, Frontenac, Lennox et Addington, le Laboratoire de Santé publique Ontario et les hôpitaux gérant les centres d'évaluation ont pu contenir et gérer une éclosion de COVID-19 dans un établissement de services personnels grâce à une communication publique continue, des inspections rapides, des tests de dépistage intensifs, des ordonnances de la santé publique, des temps d'exécution des tests de dépistage courts et une gestion approfondie des cas et des contacts dans les 24 heures suivant des résultats de laboratoire positifs. La communauté de la région de Kingston, Frontenac, Lennox et Addington a également joué un rôle crucial en se protégeant mutuellement en passant des tests de dépistage ainsi qu'en respectant les consignes du service de Santé publique de la région de Kingston, Frontenac, Lennox et Addington, à savoir de s'auto-isoler et porter des couvre-visages dans les espaces publics intérieurs. Ces actions peuvent éclairer la prise de décision d'autres compétences qui pourraient être confrontées à de futures éclosions similaires. Une approche collective et collaborative est nécessaire lors d'une éclosion, car tous les membres de la communauté doivent travailler ensemble pour limiter la propagation de la maladie.

\section{Déclaration des auteurs}

A. L. et S. P. - Rédaction du projet original

A. L. - Visualisation, analyse et interprétation des données

A. K. et T. H. G. - Enquêtes épidémiologiques et environnementales et intervention et rétroaction fournies K. M. - Supervision des enquêtes épidémiologiques et environnementales, commentaires, supervision du travail

Tous les auteurs ont contribué à la conceptualisation du manuscrit.

Le contenu de l'article et les points de vue qui y sont exprimés n'engagent que les auteurs et ne correspondent pas nécessairement à ceux du gouvernement du Canada.

\section{Intérêts concurrentss}

Aucun.

\section{Remerciements}

Les auteurs tiennent à remercier tout le personnel de Santé publique de la région de Kingston, Frontenac, Lennox et Addington qui a contribué à l'enquête sur cette éclosion, notamment les enquêteurs, les infirmières l'équipe de gestion des cas et des contacts ainsi que le personnel du centre d'évaluation et le personnel du Kingston Health Sciences Centre, le Lennox and Addington County General Hospital et le Laboratoire de Santé Ontario. Les auteurs tiennent également à remercier les personnes suivantes pour leur contribution à ce rapport : J. Sousa, A. Kern, D. Rines, C. Tran, B. Mosely, A. van Dijk et S. Biro. Enfin, les auteurs tiennent à remercier toutes les personnes et organisations touchées par cette éclosion et la pandémie de COVID-19. 


\section{Financement}

Aucun financement externe n'a été reçu.

\section{Références}

1. World Health Organization. Coronavirus disease (COVID-19) pandemic. WHO; 2019 (accédé 2020-07-24). https://www. who.int/emergencies/diseases/novel-coronavirus-2019

2. Centers for Disease Control and Prevention. Coronavirus Disease 2019 (COVID-19) - Symptoms. Centers for Disease Control and Prevention. CDC; (modifié 2020-12; accédé 2020-08-13). https://www.cdc.gov/coronavirus/2019-ncov/ symptoms-testing/symptoms.html

3. CDC COVID-19 Response Team. COVID-19 Response Team. Severe outcomes among patients with coronavirus disease 2019 (COVID-19)_United States, February 12-March 16, 2020. MMWR Morb Mortal Wkly Rep 2020;69(12):343-6. DOI PubMed

4. Hui DS, I Azhar E, Madani TA, Ntoumi F, Kock R, Dar O, Ippolito G, Mchugh TD, Memish ZA, Drosten C, Zumla A, Petersen E. The continuing 2019-nCoV epidemic threat of novel coronaviruses to global health - The latest 2019 novel coronavirus outbreak in Wuhan, China. Int J Infect Dis 2020;91:264-6. DOI PubMed

5. Règlement de l'Ontario. O. Reg. 135/18 : Désignation de maladies. https://www.ontario.ca/fr/lois/reglement/180135

6. Agence de la santé publique du Canada. Maladie à coronavirus (COVID-19). Ottawa (ON) : ASPC; (modifié 2020-02; accédé 2020-07-24). https://www.canada. $\mathrm{ca} / \mathrm{fr} / \mathrm{sante-publique/services/maladies/maladie-coronavirus-}$ covid-19.html

7. Gouvernement de l'Ontario. Ministère de finances. Mise à jour des projections démographiques pour l'Ontario, 2019-2046. Gouvernement de l'Ontario; (modifié 2020-10; accédé 2020-08-11). https://www.fin.gov.on.ca/en/economy/ demographics/projections/

8. Gouvernement de l'Ontario. Un cadre visant le déconfinement de la province. Gouvernement de l'Ontario; (modifié 2020-11; accédé 2020-08-11). https://www.ontario. $\mathrm{ca} / \mathrm{fr} /$ page/un-cadre-visant-le-deconfinement-de-la-province

9. Gouvernement de l'Ontario. Ministère de la Santé. Document d'orientation à l'intention du secteur de la santé. Gouvernement de l'Ontario; (modifié 2020-02; accédé 2020-07-24). https://www.health.gov.on.ca/fr/pro/programs/ publichealth/coronavirus/2019_guidance.aspx

10. Gouvernement de l'Ontario. Ministère de la Santé. Gestion des cas et des contacts relatifs à la COVID-19 en Ontario; (version 11.0). Gouvernement de l'Ontario; (modifié 2020-01; accédé 2020-07-30). https://www.health. gov.on.ca/fr/pro/programs/publichealth/coronavirus/docs/ contact_mngmt/management_cases_contacts.pdf
11. Gouvernement de l'Ontario. Ministère de la Santé. Mise à jour sur le document d'orientation sur la COVID-19 : Tests de dépistage provinciaux, version 110, 5 mars 2021. Gouvernement de l'Ontario; (accédé 2020-07-30). https://www.health.gov.on.ca/fr/pro/programs/publichealth/ coronavirus/docs/2019_testing_guidance.pdf

12. Huang YT, Tu YK, Lai PC. Estimation of the secondary attack rate of COVID-19 using proportional meta-analysis of nationwide contact tracing data in Taiwan. J Microbiol Immunol Infect. Infect 2021;54(1):89-92. DOI PubMed

13. Public Health Ontario. L'indice de marginalisation ontarien (ON-Marg). Toronto (ON) : SPO; (modifié 2018-10; accédé 2020-07-24). https://www.publichealthontario.ca/fr/ data-and-analysis/health-equity/ontario-marginalizationindex

14. Kingston F; Lennox and Addington Public Health. Nails and aesthetic services COVID-19 prevention checklist. KFLAPH; 2020 (accédé 2020-08-14). https://www.kflaph.ca/en/ partners-and-professionals/resources/Coronavirus/2020-0623-Nails-Aesthetic-Services-COVID-19-Prevention-Checklist. pdf

15. Gouvernement de l'Ontario. COVID-19 : résumés épidémiologiques de Santé publique Ontario. Toronto (ON) : Gouvernement de l'Ontario; (modifié 2021-02; accédé 2020-07-24). https://covid-19.ontario.ca/fr/covid19-resumes-epidemiologiques-de-sante-publique-ontario

16. Pavlik I, Falkinham JO, Kazda J. Environments providing favourable conditions for the multiplication and transmission of mycobacteria. In: Kazka J, Pavlik I, Falkinham JO, Hruska K. The Ecology of Mycobacteria: Impact on Animal's and Human's Health. Dordrecht: Springer Netherlands; 2009. p. 89-197 (accédé 2020-08-06). http://link.springer. com/10.1007/978-1-4020-9413-2_5

17. Koroglu M, Demiray T, Ozbek A, Guclu E, Karabay O, Altindis M, Durmaz R. Nail scissors and fingernails as reservoirs of hepatitis $B$ virus DNA: role of nail scissors in household transmission of hepatitis B virus. Am J Infect Control 2018;46(7):793-7. DOI PubMed

18. Yang J, Hall K, Nuriddin A, Woolard D. Risk for hepatitis B and $C$ virus transmission in nail salons and barbershops and state regulatory requirements to prevent such transmission in the United States. J Public Health Manag Pract 2014;20(6):E20-30. DOI PubMed

19. Barn P, Chen T. A narrative review of infections associated with personal service establishments Part I: aesthetics. Environ Health Rev. 2012;55(1):19-26. DOI

20. Hendrix MJ, Walde C, Findley K, Trotman R. Absence of apparent transmission of SARS-CoV-2 from two stylists after exposure at a hair salon with a universal face covering policy-Springfield, Missouri, May 2020. MMWR Morb Mortal Wkly Rep 2020;69(28):930-2. DOI PubMed

21. Mizumoto K, Kagaya K, Zarebski A, Chowell G. Estimating the asymptomatic proportion of coronavirus disease 2019 (COVID-19) cases on board the Diamond Princess cruise ship, Yokohama, Japan, 2020. Euro Surveill 2020;25(10):2000180. DOI PubMed 
31. Grijalva CG, Rolfes MA, Zhu Y, McLean HQ, Hanson KE, Belongia EA, Halasa NB, Kim A, Reed C, Fry AM, Talbot HK. Transmission of SARS-COV-2 Infections in Households Tennessee and Wisconsin, April-September 2020. MMWR Morb Mortal Wkly Rep 2020;69(44):1631-4. DOI PubMed

23. Rivett $L$, Sridhar $S$, Sparkes $D$, Routledge $M$, Jones NK, Forrest $\mathrm{S}$, Young J, Pereira-Dias J, Hamilton WL, Ferris $\mathrm{M}$, Torok ME, Meredith L, Curran MD, Fuller S, Chaudhry A, Shaw A, Samworth RJ, Bradley JR, Dougan G, Smith KG, Lehner PJ, Matheson NJ, Wright G, Goodfellow IG, Baker S, Weekes MP; CITIID-NIHR COVID-19 BioResource Collaboration. Screening of healthcare workers for SARS-CoV-2 highlights the role of asymptomatic carriage in COVID-19 transmission. eLife 2020;9:e58728. DOI PubMed

24. Arons MM, Hatfield KM, Reddy SC, Kimball A, James A, Jacobs JR, Taylor J, Spicer K, Bardossy AC, Oakley LP, Tanwar S, Dyal JW, Harney J, Chisty Z, Bell JM, Methner M, Paul P, Carlson CM, McLaughlin HP, Thornburg N, Tong S, Tamin A, Tao Y, Uehara A, Harcourt J, Clark S, Brostrom-Smith C, Page LC, Kay M, Lewis J, Montgomery P, Stone ND, Clark TA, Honein MA, Duchin JS, Jernigan JA; Public Health-Seattle and King County and CDC COVID-19 Investigation Team. Presymptomatic SARS-CoV-2 infections and transmission in a skilled nursing facility. N Engl J Med 2020;382(22):2081-90. DOI PubMed

25. Furukawa NW, Brooks JT, Sobel J. Evidence supporting transmission of severe acute respiratory syndrome coronavirus 2 while presymptomatic or asymptomatic. Emerg Infect Dis 2020;26(7):e201595. DOI PubMed

26. Park SW, Cornforth DM, Dushoff J, Weitz JS. The time scale of asymptomatic transmission affects estimates of epidemic potential in the COVID-19 outbreak. Epidemics 2020;31:100392. DOI PubMed

27. Wang Z, Ma W, Zheng X, Wu G, Zhang R. Household transmission of SARS-CoV-2. J Infect 2020;81(1):179-82. DOI PubMed

28. Jing QL, Liu MJ, Zhang ZB, Fang LQ, Yuan J, Zhang AR, Dean NE, Luo L, Ma MM, Longini I, Kenah E, Lu Y, Ma Y, Jalali N, Yang ZC, Yang Y. Household secondary attack rate of COVID-19 and associated determinants in Guangzhou, China: a retrospective cohort study. Lancet Infect Dis 2020;20(10):1141-50. DOI PubMed

29. Li W, Zhang B, Lu J, Liu S, Chang Z, Peng C, Liu X, Zhang P, Ling Y, Tao K, Chen J. Characteristics of household transmission of COVID-19. Clin Infect Dis 2020;71(8):1943-6. DOI PubMed

30. Bi Q, Wu Y, Mei S, Ye C, Zou X, Zhang Z, Liu X, Wei L, Truelove SA, Zhang T, Gao W, Cheng C, Tang X, Wu X, Wu Y, Sun B, Huang S, Sun Y, Zhang J, Ma T, Lessler J, Feng T. Epidemiology and transmission of COVID-19 in 391 cases and 1286 of their close contacts in Shenzhen, China: a retrospective cohort study. Lancet Infect Dis 2020;20(8):911-9. DOI PubMed

32. Lau JT, Lau M, Kim JH, Tsui HY, Tsang T, Wong TW. Probable secondary infections in households of SARS patients in Hong Kong. Emerg Infect Dis 2004;10(2):236-43. DOI PubMed

33. Drosten C, Meyer B, Müller MA, Corman VM, Al-Masri M, Hossain R, Madani H, Sieberg A, Bosch BJ, Lattwein E, Alhakeem RF, Assiri AM, Hajomar W, Albarrak AM, Al-Tawfiq JA, Zumla Al, Memish ZA. Transmission of MERS-coronavirus in household contacts. N Engl J Med 2014;371(9):828-35. DOI PubMed

34. Tsang TK, Lau LL, Cauchemez S, Cowling BJ. Household transmission of influenza virus. Trends Microbiol 2016;24(2):123-33. DOI PubMed

35. Sneppen K, Taylor RJ, Simonsen L. Impact of superspreaders on dissemination and mitigation of COVID-19. medRxiv. 2020.05.17.20104745. DOI

36. Hamner L, Dubbel P, Capron I, Ross A, Jordan A, Lee J, Lynn J, Ball A, Narwal S, Russell S, Patrick D, Leibrand H. High SARS-CoV-2 attack rate following exposure at a choir practice-Skagit County, Washington, March 2020. MMWR Morb Mortal Wkly Rep 2020;69(19):606-10. DOI PubMed

37. Lin J, Yan K, Zhang J, Cai T, Zheng J. A super-spreader of COVID-19 in Ningbo city in China. J Infect Public Health 2020;13(7):935-7. DOI PubMed

38. Leng T, White $C$, Hilton J, Kucharski AJ, Pellis L, Stage $\mathrm{H}$, Davies N, CMMID-Covid-19 WG, Keeling MJ, Flasche S. The effectiveness of social bubbles as part of a Covid-19 lockdown exit strategy, a modelling study. medRxiv. 2020.06.05.20123448. DOI

39. Santé publique Ontario. Résumé épidémiologique amélioré : La COVID-19 en Ontario : un regard sur la précarité économique. Toronto (ON) : SPO; 2020 (accédé 2020-08-24). https://www.publichealthontario.ca/-/ media/documents/ncov/epi/2020/06/covid-19-epi-materialdeprivation.pdf?la=fr

40. Veličko I, Ploner A, Sparén P, Marions L, Herrmann B, Kühlmann-Berenzon S. Sexual and testing behaviour associated with Chlamydia trachomatis infection: a cohort study in an STI clinic in Sweden. BMJ Open 2016;6(8):e011312. DOI PubMed

41. Chesang K, Hornston S, Muhenje O, Saliku T, Mirjahangir J, Viitanen A, Musyoki H, Awuor C, Githuka G, Bock N. Healthcare provider perspectives on managing sexually transmitted infections in HIV care settings in Kenya: A qualitative thematic analysis. PLoS Med 2017;14(12):e1002480. DOI PubMed

42. von Wyl V, Bonhoeffer S, Bugnion E, Puhan MA, Salathé $M$, Stadler T, Troncoso C, Vayena E, Low N. A research agenda for digital proximity tracing apps. Swiss Med Wkly 2020;150(2930):w20324. DOI PubMed 